\title{
A New Approach to Achieving Sharp and Timely Deliveries in Supply Chain Networks
}

\author{
Dinesh Garg \\ Indian Institute of Science \\ Bangalore - India
}

\author{
Y. Narahari \\ Indian Institute of Science \\ Bangalore - India
}

\author{
N. Viswanadham \\ The Logistics Institute Asia Pacific \\ NUS, Singapore - 119260
}

\begin{abstract}
In this paper, we come up with an innovative approach through which variability reduction and synchronization can be realized in supply chains. The approach developed is founded on a connection between mechanical design tolerancing and supply chain lead time compression. We use two metrics for delivery performance, delivery sharpness and delivery probability, which measure the accuracy as well as the precision with which products are delivered to the customers. Then we solve the following specific problem: given the delivery sharpness and delivery probability to be achieved, how can variability be allocated across individual stages of the supply chain in a cost-effective way. We call this the variance pool allocation (VPA) problem and we suggest a systematic approach for solving the VPA problem. We show that a variety of important supply chain design problems, such as supply chain partner selection, can be posed as instances of the VPA problem. We formulate and solve the VPA problem for a plastics industry supply chain and demonstrate how the solution can be used to choose the best mix of supply chain partners.
\end{abstract}

\section{Keywords}

Supply chain management, variability reduction, process capability indices, statistical tolerancing, variance pool allocation (VPA).

\section{INTRODUCTION}

The key issue in supply chain design, facing companies today, is no longer the location of manufacturing and distribution facilities for their vertically integrated operations, but rather the strategic selection of partners for each stage of their outsourced value chain, in the face of uncertainties of various kinds. This selection needs to take into account the synchronization of schedules for suppliers, manufacturers, and logistics providers in order to streamline processes throughout the supply chain. Variability is a major issue in this process. Variability reduction and business process synchronization are therefore acknowledged as key to achieving superior levels of performance in supply chain networks.

The complexity of strategic supply chain design process requires that it be supported with powerful analytical tools and models. Our paper addresses this need by coming up with a novel analytical approach to the supply chain design problem. Variability reduction is the key idea underlying our approach.
Lead time compression in supply chains is the subject of several recent papers, see for example, Garg, Narahari, and Viswanadham [1]. Statistical design tolerancing is a mature subject in the design community. The key ideas in statistical design tolerancing which provide the core inputs to this paper are: (1) theory of process capability indices [2]; (2) Motorola six sigma program [3]; and (3) design for tolerancing [4]. The present paper innovatively uses the key ideas and notions in the area of statistical design tolerancing in achieving variability reduction and synchronization in the supply chain process, leading to timely and sharp deliveries. A preliminary version of this paper [1] contains some of these above ideas. A companion paper [5] explores these ideas in a different direction and applies the ideas to inventory optimization in multi-stage supply chains.

The paper is organized in the following way. In Section 2 , we review process capability indices, $C_{p}, C_{p k}$, and $C_{p m}$ and present an innovative interpretation of these indices in the context of the supply chain process. In Section 3, we formulate the variance pool allocation (VPA) problem. We present a five stage approach for solving the VPA problem for linear supply chains (that is, supply chains with linear or pipelined workflow). In Section 4, we discuss one real-world case study. The system studied is a six stage, linear, make-to-order plastics industry supply chain in Maharashtra, India. For this supply chain, we compute the optimal way of distributing variabilities so as to achieve desired levels of DP and DS. We use these results to the best mix of service providers across the six stages of the supply chain in an optimal way.

\section{Supply Chain Process Capability Indices}

The process capability indices $C_{p}, C_{p k}$, and $C_{p m}$ [2] are popular in the areas of design tolerancing and statistical process control. For every business process which is a part of modern electronic or web enabled supply chain, delivery time of product is an important quality characteristic. Variability in lead time is inherent to almost all the business processes, therefore, it will be apt to apply the notion of process capability indices to measure the delivery capability or delivery quality of any business process. Consider logistics process in a supply chain where goods are transferred from location $P$ to location 
$Q$ through trucks. The transportation time is random in nature which is assumed to be normally distributed with mean $\mu=48$ hrs. and standard deviation 2 hrs. Let us assume that it is required that goods should not reach earlier than $36 \mathrm{hrs}$. due to limited storage space. Also, goods should reach within $60 \mathrm{hrs}$. after dispatching from $A$, otherwise potential customers will be lost at $B$. Ideal time suggested for arrival of goods at location $B$ is $48 \mathrm{hrs}$. after loading the truck from location $A$. First we analyze this model and then change some of the parameters and see how the quality of the logistic process is going to be affected.

For the lead time of the given logistics process, the known parameters are as follows.

$$
\begin{array}{rlrl}
\mu & =48 & \tau & =48 \\
\sigma=2 & U & =60 \\
& =2 & =36
\end{array}
$$

For this model, process capability indices and yields are as follows.

$$
\begin{aligned}
& C_{p}=2 \\
& C_{p k}=2 \\
& C_{p m}=2 \\
& \text { Potential }=1-1.97317 \times 10^{-9} \\
& \text { Actual Yield }=1-1.97317 \times 10^{-9}
\end{aligned}
$$

The above measurements of the process capability imply probability of the truck arriving at location $B$ within the delivery window is approximately 1 , which means that chances of defective deliveries are almost negligible. In the following model we change system parameters of the logistics process and show that index $C_{p}$ alone is not sufficient to measure the quality of deliveries.

Consider an alternative logistics service to satisfy above logistics requirement. The mean transportation time for this service is a bit higher, i.e. 51 hrs. but the standard deviation is the same i.e. 2 hrs. For this alternative logistics service, capability indices and yields take the following values.

$$
\begin{aligned}
& C_{p}=2 \\
& C_{p k}=1.5 \\
& C_{p m}=1.1094 \text { Actual Yield }=1-3.39767 \times 10^{-6}
\end{aligned}
$$

It is easy to see that the probability of defective deliveries in this service is higher than in the previous case but $C_{p}$ is the same for both. Therefore, $C_{p}$ is not sufficient to. measure the quality of deliveries. Index $C_{p k}$ is also required along with this. Knowing both $C_{p}$ and $C_{p k}$, we can compute the probability of defective delivery. However, the next model shows that even $C_{p}$ and $C_{p k}$ put together are also not sufficient. Index $C_{p m}$ is needed to measure the quality of delivery process in a complete sense.

Case 3. The pair $\left(C_{p}, C_{p k}\right)$ is also not sufficient
Let us compare two alternative logistics services $A$ and $B$. The logistics requirement is the same as above but the mean and variance of two alternative services are as follows.

$$
\begin{aligned}
& \mu_{A}=48 \mathrm{hrs} \mu_{B}=46.4541 \mathrm{hrs} \\
& \sigma_{A}=2.5833 \mathrm{bss}=2.3231 \mathrm{hrs}
\end{aligned}
$$

For these two alternative systems process capability indices and yields come out to be as follows ( $\mathrm{P}$ stands for Potential and AY stands for actual yield).

$$
\begin{array}{rlrl}
C_{p_{A}} & =1.5484 & C_{p_{B}} & =1.7218 \\
C_{p k_{A}} & =1.5484 & C_{p k_{B}} & =1.50 \\
C_{p m_{A}} & =1.5484 & C_{p m_{B}} & =1.433 \\
\mathrm{P} & =1-3.399 \times 10^{-6} \mathrm{P} & =1-2.398 \times 10^{-7} \\
\mathrm{AY} & =1-3.399 \times 10^{-6} \mathrm{AY} & =1-3.399 \times 10^{-6}
\end{array}
$$

In these two alternative systems, even though pair $\left(C_{p}\right.$, $\left.C_{p k}\right)$ is different, the probability of defective deliveries is the same. Therefore, the pair $\left(C_{p}, C_{p k}\right)$ which completely decides the probability of defective deliveries, is not sufficient to completely quantify the quality of a process. Here $C_{p m}$ is different for the two logistics service providers. Logistics service $A$ is better than logistics service $B$ because in service $A$ most of the deliveries are close to target, i.e. 48 hrs.

\section{A. Delivery Probability and Delivery Sharpness}

The above example suggests that the 3-tuple $\left(C_{p}, C_{p k}, C_{p m}\right)$ is sufficient to measure the delivery quality of any business process in a given supply chain. Because a unique $\left(C_{p}, C_{p k}\right)$ pair has a unique actual yield, the 3-tuple $\left(C_{p}, C_{p k}, C_{p m}\right)$ can be substituted by the pair (Actual yield, $C_{p m}$ ) to measure the delivery quality. Being an indicator for precision and accuracy of the deliveries, we prefer to call actual yield of the process as Delivery Probability $(D P)$ and $C_{p m}$ as Delivery Sharpness $(D S)$. In the present paper, we use these two indices to measure the quality of any delivery process in a given supply chain. Rather than expressing the DP in terms of numerical values, we prefer to express it in terms of $\theta \sigma$ levels where $\theta \in \mathfrak{R}^{+}$. Each $\theta \sigma$ level corresponds to a unique number in the interval $[0,1]$.

We are motivated by the Motorola six sigma (MSS) program [?] in using the idea of $\theta \sigma$ levels. In the MSS program, a unique $\sigma$ level is attached to a unique number in the interval $[0,1]$. In the MSS program, these numbers corresponds to upper bounds on the yield of the process but here we assume that these numbers correspond to the actual yield of the process. For example, according to the MSS program, in the presence of process mean shifts and drifts, if upper bound on yield of the process is equal to $1-3.4 \times 10^{-6}$ then its quality is $6 \sigma$ quality. In the framework of DP and DS, we call a process DP is $6 \sigma$ iff its actual yield is $1-3.4 \times 10^{-6}$. Moreover, in the framework 
of DP and DS no shifts and drifts are allowed in process mean, only bias is allowed between process mean and target value.

\section{VARIANCE POOL Allocation PROBlem}

Consider a linear, make-to-order (MTO) supply chain with $n$ stages. This supply chain is a single product supply chain. The product is delivered to the end customer from stage $n$. In the present model we are not concerned about how the orders are consolidated, how the production planning is done, and at which intermediate stages finished goods or semi-finished goods inventory will be maintained. Let us assume that as soon as any customer places an order for a unit of the product, the flow of material against the order starts from stage 1 , undergoes processing at successive stages, and is finally delivered to the customer after processing at stage $n$. Let the lead time at each stage be considered as a continuous random variable (i.e. $X_{i}, i=1,2, \ldots, n$ ). As a consequence of this assumption, the end- to-end lead time is also a continuous random variable.

The first objective of the study here is to find out how the variance of lead time of individual stages should be chosen, assuming that the mean lead time is given for each stage, such that the specified levels of DP and DS are attained for a specified end-to-end lead time delivery window in a cost effective way. The second objective is to show a compelling application of the above; as an example, we show how the above can be used in choosing the best possible mix of alternatives for supply chain operation. For the specific problem considered, we describe below how we move from the first objective to the second objective.

\section{A. Assumptions}

The model for VPA, proposed in this paper, is based upon the following assumptions on the nature of the business process and the customer delivery window:

1) Lead time $X_{i}$ at stage $i(i=1, \ldots, n)$ is normally distributed, say, with mean $\mu_{i}$ and standard deviation $\sigma_{i}$. Also individual lead times are mutually independent.

2) There is no time elapsed between end of process $i$ to commencement of process $i+1(i=1, \ldots, n-1)$. This is a reasonable assumption since any interface time can be absorbed into the lead time of stage $i$ or lead time of stage $i+1$. As an immediate consequence of this assumption, the end-to-end lead time, $Y$, is equal to the sum of lead times of the individual processes. $Y$ can be easily seen to be normally distributed with $\mu=\sum_{i=1}^{n} \mu_{i}$ and $\sigma^{2}=$ $\sum_{i=1}^{n} \sigma_{i}^{2}$.

3) Each customer specifies a customer delivery window $(\tau, T)$ where $\tau$ is the desirable amount of the time a customer is willing to wait after placing the order. The customer is prepared to wait for a maximum period of $\tau+T$. Also, the customer does not want the delivery to occur before $\tau-T$. Therefore, $\tau$ is the target value for end-to-end delivery process and $T$ is the tolerance.

\section{B. Formulation of the VPA Problem}

1) Known Parameters: The following parameters are known in a typical VPA problem. (1) End customer delivery window for end-to-end lead time $(\tau, T)$.

(2) Mean $\mu_{i}$ of random variable $X_{i}, i=1,2, \ldots, n$.

(3) Delivery Probability and Delivery Sharpness for end-toend lead time, $Y$.

(4) Processing cost per unit product at each stage $i$, denoted by $\mathscr{K}_{i}$, of the supply chain. This cost is the part of the total processing cost that is associated with lead time. Assume that variance $\sigma_{i A}, \sigma_{i B}, \ldots$ of processing time and per unit processing $\operatorname{cost} C_{i A}, C_{i B}, \ldots$ is available for each one of the service providers $A, B, \ldots$ for stage $i$. These pairs $\left(\sigma_{i A}, C_{i A}\right),\left(\sigma_{i B}, C_{i B}\right), \ldots$ can be used to get a polynomial function for per unit processing cost in terms of $\sigma_{i}$. For the sake of conceptual and computational simplicity we are motivated behind choosing a second order polynomial in the following manner:

$$
\mathscr{K}_{i}=A_{i 0}+A_{i 1} \sigma_{i}+A_{i 2} \sigma_{i}^{2}
$$

Here $A_{i 0}, A_{i 1}, A_{i 2}$ are constants and not all are positive. These constants will be obtained by polynomial curve fitting for the pairs $\left(\sigma_{i A}, C_{i A}\right),\left(\sigma_{i B}, C_{i B}\right), \ldots$.

2) Decision Variables: The decision variables of the VPA problem are $P_{1}^{*}, P_{2}^{*}, \ldots, P_{n}^{*}$ where $P_{i}^{*}$ denotes one of the partner (or service provider) out of a available set of partners for stage $i$. As we mentioned the scheme earlier to get this set, we will first compute optimal standard deviations $\sigma_{i}^{*}$ of each individual stage $i(i=1, \ldots, n)$ and then use them to get the final result in terms of the set $P_{1}^{*}, P_{2}^{*}, \ldots, P_{n}^{*}$. This is the reason why the problem is called as variance pool allocation.

3) Objective Function and Constraints : In the present model, we have confined our discussion only to lead time variability of the supply chain without considering other issues like demand variability, inventory levels, etc. Therefore, it seems to be reasonable to consider the total processing cost of a single unit of product, denoted by $\mathscr{K}$, as the objective function. This cost is simply the sum of processing costs of all the stages. Thus the problem formulation becomes:

Minimize

$$
\mathscr{K}=\sum_{i=1}^{n} \mathscr{K}_{i}=\sum_{i=1}^{n}\left(A_{i 0}+A_{i 1} \sigma_{i}+A_{i 2} \sigma_{i}^{2}\right)
$$

subject to

$$
\begin{aligned}
\text { DS for end-to-end lead time } & \geq C_{p m}^{*}(3) \\
\text { DP for end-to-end lead time } & \geq 6 \sigma \text { (4) } \\
\sigma_{i} & >0 \forall i(5)
\end{aligned}
$$




\begin{tabular}{|c|c|c|c|c|c|c|}
\hline Stage & \multicolumn{6}{|c|}{ Service Providers } \\
\hline $\bar{i}$ & \multicolumn{2}{|c|}{$\bar{A}$} & \multicolumn{2}{|c|}{$\bar{B}$} & \multicolumn{2}{|c|}{$\mathrm{C}$} \\
\hline & $\begin{array}{c}\sigma_{A i} \\
\text { (days) }\end{array}$ & $\begin{array}{c}\bar{C}_{A i} \\
\text { (\$/item) }\end{array}$ & $\begin{array}{c}\sigma_{B i} \\
\text { (days) }\end{array}$ & $\begin{array}{c}C_{B i} \\
(\$ / \text { item })\end{array}$ & $\begin{array}{c}\sigma_{C i} \\
\text { (days) }\end{array}$ & $\underset{\text { (\$fitem) }}{C_{C i}}$ \\
\hline 1 & 0.50 & 256.18 & 0.75 & 161.44 & 1.00 & 125.69 \\
\hline 2 & $0 . \overline{40}$ & 436.40 & 0.50 & 413.03 & 0.60 & 390.37 \\
\hline 3 & 0.10 & 077.79 & 0.20 & 049.33 & 0.30 & 034.8 \\
\hline 4 & 0.40 & 436.40 & 0.50 & 413.03 & 0.60 & 390.37 \\
\hline 5 & 0.50 & 185.79 & 1.00 & 080.34 & 1.50 & 065.27 \\
\hline 6 & 0.10 & 077.79 & 0.20 & 049.33 & 0.30 & 034.81 \\
\hline
\end{tabular}

TABLE I

STANDARD DEVIATION OF LEAD TIMES AND COST FOR EACH SERVICE PROVIDER

\section{Solution Approach}

We outline a a 5-Step procedure for formulating and solving the VPA problem.

1) Problem Formulation

2) Expressing the Constraints in terms of Decision Variables

3) Determining the optimal values of $C_{p}$ and $C_{p k}$

4) Solving the optimization problem

5) Solving a specific design optimization problem

\section{A Plastics Industry Case Study}

We now consider a supply chain for a plastics industry and apply the 5-Step approach for formulating and solving the VPA problem. The supply chain has six business processes namely (1) Procurement, (2) Sheet Fabrication, (3) Inbound Logistics, (4) Manufacturing, (5) Assembly, and (6) Outbound Logistics. Let all the business processes in the supply chain satisfy the assumptions mentioned in Section III-A. Assume for the sake of convenience that there are three alternatives (call them service providers) at each of the six stages. The problem here is to determine the optimal mix of service providers for each stage such that the end -to end delivery probability is at least at $6 \sigma$ level and delivery sharpness is at least, say, 1.4. Suppose for each stage, the mean lead time for all the three alternative service providers is same. Let the mean $\mu_{i}$ for $i=1,2,3,4,5$ and 6 be 7 days, 30 days, 3 days, 30 days, 10 days, and 3 days respectively. Let the target value of supply chain lead time $Y$ be 82 days and tolerance be 6.5 days. This implies: $\tau=82$ days and $T=6.5$ days. The processing cost of one unit of product at each one of the six business processes, varies over the service providers as a function of variance of lead times. Table I gives the values of per unit processing cost and processing time variance for each service provider. Thus this problem is a VPA problem which can be solved as follows.

\section{A. Step 1}

Some of the known parameters for VPA problem are provided explicitly in the given problem. The parameters

\begin{tabular}{|c|c|c|c|}
\hline Stage & 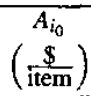 & $\begin{array}{c}\bar{A}_{i_{1}} \\
\left(\frac{\$}{\text { item-day }}\right)\end{array}$ & $\left(\frac{A_{i_{2}}}{\$} \frac{\$}{\text { item-day }}\right.$ \\
\hline Procurement & 622.634 & -968.872 & 471.928 \\
\hline Sheet Fabrication & 537.011 & -265.752 & 035.604 \\
\hline Inbound Logistics & 120.186 & .493 .651 & 696.919 \\
\hline Manufacturing & 537.011 & -265.752 & 035.604 \\
\hline Assembly & 381.625 & -482.053 & 180.770 \\
\hline Outbound Logistics & 120.186 & -493.651 & 696.919 \\
\hline
\end{tabular}

TABLE II

COST COEFFICIENTS FOR PLASTIC INDUSTRY SLPPLY CHAIN PROBLEM

which will be needed in further calculations and are implicit to the problem are $\mu, d, \theta, C_{p m}^{*}$, and $A_{i j}$. If we denote lead time distribution of Procurement, Sheet Fabrication, Inbound Logistics, Manufacturing, Assembly, and Outbound Logistics by $X_{1}, X_{2}, X_{3}, X_{4}, X_{5}$, and $X_{6}$ respectively, then it is easy to see that

$$
\begin{aligned}
\mu & =\sum_{i=1}^{6} \mu_{i}=83 \text { days } \\
d & =\min (\tau+T-\mu, \mu-\tau+T)=5.5 \text { days } \\
\theta & =6 \\
C_{p m}^{*} & =1.4
\end{aligned}
$$

The coefficients $A_{i j}$ can be obtained by a second order polynomial curve fitting for the given three pairs () for each stage $i$. The coefficients $A_{i j}$ obtained by such an approximation are tabulated in Table II. Now the optimization problem can be formulated as follows:

Minimize

$$
\mathscr{K}=\sum_{i=1}^{6} \mathscr{X}_{i}=\sum_{i=1}^{6}\left(A_{i 0}+A_{i 1} \sigma_{i}+A_{i 2} \sigma_{i}^{2}\right)
$$

subject to

$$
\begin{aligned}
\text { DS for end-to-end lead time } & \geq 1.4 \\
\text { DP for end-to-end lead time } & \geq 6 \sigma \\
\sigma_{i} & >0, i=1,2, \ldots, 6
\end{aligned}
$$

where $A_{i_{0}}, A_{i_{1}}$, and $A_{i_{2}}$, are provided in Table II.

\section{B. Step 2}

The constraints of the optimization problem presented in Step 1 can be expressed in terms of decision variables as:

$$
\begin{aligned}
\sum_{i=1}^{6} \sigma_{i}^{2} & =\frac{42.25}{9 C_{p}^{*^{2}}}=\frac{30.25}{9 C_{p k}^{*^{2}}} \\
\sigma_{i} & >0 \forall i=1,2, \ldots, 6
\end{aligned}
$$




\section{Step 3}

It is easy to see $\overline{C_{p m}}$ for this problem is 2.1667 which is greater than 1.4. Therefore, the problem is feasible. As a next step, we solve the corresponding unconstrained optimization problem and get the point $\left(C_{p}^{g}, C_{p k}^{g}\right)$ which leads to global minimum cost and then test whether this point falls into feasible region or not.

For this, let $S=\left\{\left(\sigma_{1}, \sigma_{2}, \sigma_{3}, \sigma_{4}, \sigma_{5}, \sigma_{6}\right): \sigma_{i} \in \Re^{+} \forall i=\right.$ $1,2,3,4,5,6\}$. It immediately follows from this definition of $S$ that $\mathscr{K}: S \rightarrow E_{1}$ where $S$ is a nonempty open convex set. Moreover, for testing the convexity of function $\mathscr{K}$, we compute the gradient vector and Hessian matrix of the function. Note that the gradient vector $\nabla \mathscr{K}(\overline{\mathbf{X}})$ for function $\mathscr{K}$ at point $\overline{\mathbf{X}}=\left(\sigma_{1}, \sigma_{2}, \sigma_{3}, \sigma_{4}, \sigma_{5}, \sigma_{6}\right)^{T}$ can be obtained as

$$
\nabla \mathscr{K}(\overline{\mathbf{X}})=\left[\begin{array}{c}
\frac{\partial \mathscr{X}(\overline{\mathbf{X}})}{\partial \sigma_{1}} \\
\frac{\partial \mathscr{X}(\overline{\mathbf{X}})}{\partial \sigma_{2}} \\
\frac{\partial \mathscr{X}(\overline{\mathbf{X}})}{\partial \sigma_{3}} \\
\frac{\partial \mathscr{X}(\overline{\mathbf{X}})}{\partial \sigma_{4}} \\
\frac{\partial \mathscr{K}(\overline{\mathbf{X}})}{\partial \sigma_{5}} \\
\frac{\partial \mathscr{X}(\overline{\mathbf{X}})}{\partial \sigma_{6}}
\end{array}\right]=\left[\begin{array}{c}
A_{11}+2 A_{12} \sigma_{1} \\
A_{21}+2 A_{22} \sigma_{1} \\
A_{31}+2 A_{32} \sigma_{1} \\
A_{41}+2 A_{42} \sigma_{1} \\
A_{51}+2 A_{52} \sigma_{1} \\
A_{61}+2 A_{62} \sigma_{1}
\end{array}\right]
$$

Also, the Hessian $H(\overline{\mathbf{X}})$ for function $\mathscr{K}$ at point $\overline{\mathbf{X}}=$ $\left(\sigma_{1}, \sigma_{2}, \sigma_{3}, \sigma_{4}, \sigma_{5}, \sigma_{6}\right)^{T}$ can be obtained as

$$
H(\overline{\mathbf{X}})=\left[\begin{array}{cccccc}
2 A_{12} & 0 & 0 & 0 & 0 & 0 \\
0 & 2 A_{22} & 0 & 0 & 0 & 0 \\
0 & 0 & 2 A_{32} & 0 & 0 & 0 \\
0 & 0 & 0 & 2 A_{42} & 0 & 0 \\
0 & 0 & 0 & 0 & 2 A_{42} & 0 \\
0 & 0 & 0 & 0 & 0 & 2 A_{42}
\end{array}\right]
$$

Observe that the gradient vector and Hessian exist for each $\overline{\mathbf{X}} \in S$. Hence function $\mathscr{K}$ is twice differentiable over open convex set $S$. Moreover, the Hessian is independent of $\overline{\mathbf{X}}$. Therefore, it is enough that we test the Positive Definiteness (PD) or Positive Semi Definiteness (PSD) of Hessian at any point of $S$ instead of testing it over whole $S$.

It is easy to see that all the diagonal elements of Hessian are positive real numbers because $A_{i 2}$ are positive real numbers. Therefore, the Hessian is PD and the function $\mathscr{K}$ is strictly convex. It implies that a local minimum of function $\mathscr{K}$ is the unique global minimum. This can be obtained by equating $\nabla \mathscr{K}(\overline{\mathbf{X}})$ to 0 . The optimal values of variance for the stages come out be $\sigma_{1}^{g}=1.0265$ days, $\sigma_{2}^{g}=3.732$ days, $\sigma_{3}^{g}=0.3541$ days, $\sigma_{4}^{g}=3.732$ days, $\sigma_{5}^{g}=1.3333$ days and $\sigma_{6}^{g}=0.3541$ days. The corresponding variance of end-to-end lead time $Y$ comes out to be $\sigma^{g}=5.5622$ days. Also $C_{p}^{g}=0.3895$ and $C_{p k}^{g}=0.3296$. In order to test the feasibility of this point $\left(C_{p}^{g}, C_{p k}^{g}\right)$ first we compute the DP and DS values which will be obtained if this point is chosen as design point. The $C_{p m}$ curve and $\sigma$ curve which pass through it are those desired DP and DS. These values comes out to be $\mathrm{DP}=2.17393 \sigma$ and DS $=0.383359$.

Because these values are less than what are desired i.e. $\mathrm{DP}=6 \sigma$ and $\mathrm{DS}=1.4$, the point $\left(C_{p}^{g}, C_{p k}^{g}\right)$ cannot be taken as design point and we will have to use point $E$ (the point of intersection of the line $O P$ and feasible region) as design point. This point comes out to be:

$$
\begin{aligned}
C_{p}^{*} & =1.834364282 \\
C_{p k}^{*} & =1.552154393
\end{aligned}
$$

The DP and DS which are obtained for $Y$ by using this point as design point are $6.15645 \sigma$ and 1.4 respectively.

\section{Step 4}

Substituting the values of $C_{p}^{*}, C_{p k}^{*}$, we obtain the following constraint to work with while solving the optimization problem.

$$
\sum_{i=1}^{6} \sigma_{i}^{2}=1.389060165
$$

Now we will apply the Lagrange multiplier method to solve this optimization problem.

1. Lagrange Function

Lagrange function $L\left(\sigma_{1}, \sigma_{2}, \ldots, \sigma_{6}, \lambda\right)$ is given as:

$$
L\left(\sigma_{1}, \sigma_{2}, \ldots, \sigma_{6}, \lambda\right)=\mathscr{K}+\lambda\left(\sum_{i=1}^{6} \sigma_{i}^{2}-1.389\right)
$$

\section{Necessary Condition for Stationary Points}

Let point $\mathscr{P}^{*}=\left(\sigma_{1}^{*}, \sigma_{2}^{*}, \ldots, \sigma_{6}^{*}, \lambda^{*}\right)$ correspond to the optimal point, then this point must satisfy the following necessary conditions: 


\begin{tabular}{|c||c|c|}
\hline Stage (i) & $L_{i}$ & $R_{\mathrm{i}}$ \\
\hline 1 & $A$ & $B$ \\
\hline 2 & $A$ & $B$ \\
\hline 3 & $B$ & $C$ \\
\hline 4 & $A$ & $B$ \\
\hline 5 & $A$ & $B$ \\
\hline 6 & $B$ & $\bar{C}$ \\
\hline
\end{tabular}

TABLE III

PAIRS OF ALMOST OPTIMAL SERVICE PROVIDERS FOR EACH STAGE

$$
\begin{aligned}
& -968.872+2(471.928) \sigma_{1}^{*}+2 \lambda^{*} \sigma_{1}^{*}=0 \\
& -265.752+2(035.604) \sigma_{2}^{*}+2 \lambda^{*} \sigma_{2}^{*}=0 \\
& -493.651+2(696.919) \sigma_{3}^{*}+2 \lambda^{*} \sigma_{3}^{*}=0 \\
& -265.752+2(035.604) \sigma_{4}^{*}+2 \lambda^{*} \sigma_{4}^{*}=0 \\
& -482.053+2(180.770) \sigma_{5}^{*}+2 \lambda^{*} \sigma_{5}^{*}=0 \\
& -493.651+2(696.919) \sigma_{6}^{*}+2 \lambda^{*} \sigma_{6}^{*}=0 \\
& \sigma_{1}^{* 2}+\sigma_{2}^{* 2}+\sigma_{3}^{* 2}+\sigma_{4}^{* 2}+\sigma_{5}^{* 2}+\sigma_{6}^{* 2}=1.389
\end{aligned}
$$

Solving this system of equations by standard numerical methods we get the following solutions:

$$
\begin{aligned}
\sigma_{1}^{*} & =0.680498 \text { days } \\
\sigma_{2}^{*}=\sigma_{4}^{*} & =0.482201 \text { days } \\
\sigma_{3}^{*}=\sigma_{6}^{*} & =0.263456 \text { days } \\
\sigma_{5}^{*} & =0.572881 \text { days }
\end{aligned}
$$

Under this operating condition, the cost of delivery is:

$$
\mathscr{K}^{*}=802.299 \frac{\$}{\text { item }}
$$

It can be verified easily by the sufficiency condition that this point indeed corresponds to the point of minima.

\section{E. Step 5}

By comparing the optimal standard deviations $\sigma_{i}^{*}$ obtained in Step 4 with the given data in Table I we can compute, for each stage, the service providers whose variance is closest to the optimal. These are listed in Table III. It is easy to see from Table III that from out of these service providers, we can construct 64 combinations, with each combination representing a particular mix of service providers. We have computed the end-to-end supply chain cost $\mathscr{K}$, process capability indices $C_{p}$ and $C_{p k}, \mathrm{DP}$, and DS for each combination. It is found that the combination BBBBAB ensures the desired DP and DS level with minimum possible cost.

\section{SUMMARY AND FUTURE WORK}

In this paper, we have presented a novel approach to achieve variability reduction, synchronization, and therefore delivery performance improvement in supply chain networks. Our approach exploits connections between design tolerancing in mechanical assemblies and lead time compression in supply chain networks. The specific problem we solved here is the variance pool allocation problem. The VPA problem distributes a pool of variance across individual stages of a supply chain in a cost effective way, so as to achieve desired levels of delivery performance.

The paper leaves plenty of room for further work in several directions. The VPA problem has been investigated only for linear supply chains. Apart from computational reasons, there is no major difficulty in solving the VPA problem for supply chains with non-linear flows. In a companion article [5], we have looked at an inventory allocation problem in a multi-echelon supply chain, where we use the framework (variance pool allocation) developed in this paper to determine optimal inventory levels in different supply chain stages.

\section{REFERENCES}

[1] D. Garg, Y. Narahari, and N. Viswanadham, "Achieving sharp deliveries in supply chains through variance pool allocation," in IEEE International Conference on Robotics and Automation, ICRA-2002, Washington DC, May 2002.

[2] V. E. Kane, "Process capability indices.", Journal of Quality Technology, vol. 18, pp. 41-52, 1986.

[3] M. J. Harry and R. Stewart, "Six sigma mechanical design tolerancing," Tech. Rep., Motorola Inc., Motorola University Press, Schaumburg, IL 60196-1097, 1988.

[4] Y. Narahari, R. Sudarsan, K.W. Lyons, M.R. Duffey, and R.D. Sriram, "Design for tolerancing of electro-mechanical assemblies: An integrated approach," IEEE Transactions on Robotics \& Automation, vol. 15, no. 6, pp. 1062-1079, 1999.

[5] D. Garg, Y. Narahari, and N. Viswanadham, "Design of six sigma supply chains," in IEEE International Conference on Robotics and Automation, ICRA-2003, Taipei, May 2003. 